\title{
Optimización del diagnóstico de hepatitis por citomegalovirus en receptores de trasplante hepático: diez años de experiencia
}

\author{
Cytomegalovirus hepatitis diagnosis optimization in liver transplant recipients: \\ 10 years of experience
}

Jimena Prieto1,3, Ana Masllorens', Gonzalo Ardao1, Viviana Machado², Martin López', Solange Gerona y Julio Medina ${ }^{1,3}$

\author{
1Programa Nacional de Trasplante Hepático, Uruguay. \\ ${ }^{2}$ Hospital Militar, Uruguay. \\ ${ }^{3}$ Universidad de la República.
}

Recursos habituales disponibles, sin financiamiento externo.

Los autores no presentan conflictos de interés.

Recibido: 6 de febrero de 2020 / Aceptado: 24 de agosto de 2020

\section{Resumen}

Introducción: Para los pacientes receptores de trasplante hepático (TH) la hepatitis por citomegalovirus (CMV) constituye una entidad de difícil diagnóstico. Nuestro objetivo fue determinar la real incidencia de hepatitis por CMV aplicando técnicas diagnósticas más específicas. Material y Métodos: Estudio retrospectivo/ prospectivo, en un centro de trasplante hepático. Período de estudio: años 2009 al 2019. Se incluyeron los TH que presentaron elementos sugestivos y/o específicos de CMV en la histopatología de la punción biopsia hepática $(\mathrm{PBH})$, a los que se les realizó inmunohistoquimica (IHQ) en la PBH. Población control $n=17$. Resultados: 41 casos cumplieron los criterios de inclusión. La IHQ fue positiva en $n=6(14,6 \%)$. En la población control, la IHQ fue negativa en el $100 \%$ de los casos. Esto traduce un valor predictor negativo de $100 \%$ para la histopatología en el diagnóstico de hepatitis por CMV, con un valor predictor positivo de $14,6 \%$. En $85 \%$ de los pacientes con IHQ negativa, hubo diagnósticos alternativos. La terapia antiviral en la fase retrospectiva se indicó en $48 \%$ y en la prospectiva en $21 \%$. Conclusiones: Combinar la histopatología con la IHQ optimiza el diagnóstico de hepatitis por CMV; lo que permite la racionalización del uso de antivirales de alto costo y la búsqueda de etiologías diferenciales.

Palabras clave: trasplante hepático; citomegalovirus; hepatitis; histopatología; inmunohistoquímica.

\begin{abstract}
Background: Cytomegalovirus (CMV) hepatitis constitutes a challenging diagnostic entity in liver transplant (LT) recipients. Aim: To determine the real incidence of CMV hepatitis using more specific diagnostic tools as those currently used before. Methods: Retrospective/prospective study conducted in a hepatic transplant unit from 2009 to 2019. LT recipients with CMV specific or suggestive elements in histopathology of hepatic biopsies were included. Immunohistochemistry (IHQ) was performed in tissue samples of the studied cohort as well as in a control one. Results: 41 patients met the inclusion criteria. IHQ was diagnostic in $6(14.6 \%)$, and was negative in $100 \%$ of the control population. The negative predictive value of the histopathology for CMV hepatitis diagnosis was $100 \%$ and the positive predictive value was $14.6 \% .85 \%$ of patients in whom the IHQ was negative had alternative diagnosis Antiviral therapy in the retrospective analysis was indicated in $48 \%$ of patients and in $21 \%$ of the prospectively analyzed cohort. Conclusions: Histopathology and IHQ combination improves the diagnostic accuracy of CMV hepatitis which translates into a rational us of expensive antiviral therapy and to search for differential diagnosis

Keywords: liver transplant; cytomegalovirus; hepatitis; histopathology; immunohistochemistry.
\end{abstract}

\section{Correspondencia a:}

Jimena Prieto

jimeprieto78@gmail.com 


\section{Introducción}

$\mathrm{C}$ itomegalovirus (CMV) es un virus de la familia Herpesviridae, ubicuo y de distribución universal. $\mathrm{Al}$ igual que todos los herpesvirus, CMV establece una infección latente en el hospedero tras la recuperación de la infección aguda. La reactivación a partir de dicho estado latente puede producirse en pacientes inmunodeprimidos; ejemplo de ello son los receptores de trasplante de órgano sólidos $(\mathrm{TOS})^{1-10}$. La infección por CMV constituye una causa mayor de morbimortalidad en los receptores de TOS, con una incidencia reportada entre 30 y $80 \% \%^{2,11-18}$.

La presentación clínica de la infección por CMV se divide en: infección (viremia) asintomática y enfermedad por CMV; esta última se subdivide a su vez en síndrome viral y enfermedad con compromiso visceral (hepatitis, neumonitis, colitis), siendo esta forma la que determina una importante morbilidad ${ }^{2-19-28}$.

En receptores de trasplante hepático $(\mathrm{TH})$, la hepatitis por $\mathrm{CMV}$ es un problema, tanto por su frecuencia con una incidencia reportada de hasta $17 \%$, como por las dificultades en la realización de un diagnóstico certero ${ }^{10,11,19,29-33}$. En la mayoría de los centros de Latinoamérica los criterios utilizados para el diagnóstico de hepatitis por CMV son los de hepatitis probable; con la presencia de viremia en sangre periférica (detectada mediante antigenemia o carga viral de CMV) y el hallazgo en la histopatología de elementos sugestivos pero no específicos de infección por $\mathrm{CMV}^{32}$. La falta de realización de técnicas diagnósticas específicas determina un sobre-diagnóstico de esta entidad $y$, por ende, la instauración de terapias dirigidas, con el consiguiente gasto en salud y toxicidad adicional para el paciente. En el año 2017, nuestro grupo elaboró una encuesta online a diferentes centros latinoamericanos dirigida al diagnóstico de hepatitis por $\mathrm{CMV} ; 10$ centros respondieron (Argentina, Colombia, Paraguay, Perú, Uruguay, Costa Rica, Brasil $(\mathrm{n}=2)$, Ecuador, México); la incidencia reportada varío de menos de $10 \%$ a más de $20 \%$. El algoritmo diagnóstico utilizado lleva a que 50\% de los centros realicen punción-biopsia hepática $(\mathrm{PBH})$ de manera sistemática para el diagnóstico de hepatitis por $\mathrm{CMV}$, mientras que otro $50 \%$ solo realiza $\mathrm{PBH}$ en algunas ocasiones. Esto conlleva a muchos diagnósticos de hepatitis por CMV probable pero no confirmados ${ }^{32}$.

Actualmente existe una nueva definición de hepatitis por CMV propuesta por Ljugman y cols. ${ }^{33}$. La misma plantea como criterio necesario para realizar diagnóstico la detección del CMV en el tejido hepático mediante histopatología (inclusiones intranucleares), inmunohistoquímica (IHQ), hibridación in situ por ADN o cultivo rápido. La IHQ constituye una técnica accesible que aumenta fundamentalmente la especificidad diagnóstica en comparación con la histopatología aislada.
El objetivo del presente trabajo fue evaluar la incidencia real de hepatitis por CMV en nuestro centro mediante la aplicación de técnicas diagnósticas más específicas como la IHQ, lo que permitiría mejorar la certeza diagnóstica y optimizar el tratamiento de los pacientes.

\section{Material y Métodos}

\section{Estrategia}

Estudio analítico, que incluyó dos fases, retrospectivo/ prospectivo, en un único centro de trasplante hepático, en Montevideo, Uruguay. Los pacientes fueron incluidos prospectivamente en nuestra base de datos nacional BaDaInTOS. El período prospectivo se extendió desde el 1 de mayo de 2017 al 1 de mayo de 2019. El análisis retrospectivo se realizó desde el 14 de julio de 2009 (momento en el que comenzó el programa de trasplante) al 31 de abril de 2017.

\section{Criterios de inclusión}

Se incluyeron todos los receptores de TH que por presentar alteraciones en el hepatograma se les realizo PBH y que presentaron elementos sugestivos y/o específicos de CMV en la histopatología de la punción biopsia hepática $(\mathrm{PBH})$. A todos estos casos se les realizó IHQ en la PBH.

\section{Criterio de exclusión}

Fueron excluidos los receptores de trasplante hepático que fallecieron antes del mes post trasplante.

\section{Población control}

Estuvo constituida por receptores de $\mathrm{TH}$ en quienes se realizó $\mathrm{PBH}$ y que no presentaron elementos sugestivos ni específicos en la histopatología de hepatitis por CMV. En estos casos también se realizó IHQ en la PBH.

El diagnóstico definitivo de hepatitis por CMV confirmado se realizó con la IHQ consistente con infección por este virus. Estos pacientes se trataron con antivirales hasta tener dos resultados de carga viral de CMV en periferia indetectables por dos semanas consecutivas.

\section{PBH}

Efectuada mediante punción guiada por ecografía.

\section{Histopatología con tinción con hematoxilina/ eosina $(H \& E)$}

Fueron elementos considerados sugestivos de CMV en la histopatología la identificación de microabscesos, microgranulomas, granulomas, alteraciones citopáticas e infiltrado polimorfonuclear. Se consideró específico de $\mathrm{CMV}$ en la histopatología las inclusiones intranucleares pigmentadas o no (ojo de búho). 


\section{Inmunohistoquímica}

Procedimiento técnico basado en la utilización de anticuerpos del tipo inmunoglobulina $\mathrm{G}$, que permiten identificar marcadores antigénicos en los tejidos previamente fijados en formol tampón e incluidos en parafina. Para este estudio se utilizó el anticuerpo concentrado de ratón, monoclonal CMV, Clonas: CCH2+DDG9. Dako. Dinamarca. Codigo:M0854, realizando la técnica de inmunohistoquímica según el protocolo estandarizado por el laboratorio de Anatomía Patológica del Servicio del Hospital Militar, y respaldado en las instrucciones del datasheet correspondiente a este anticuerpo.

Se utilizaron casos controles positivos para validar la técnica en cada procedimiento realizado, asegurándose así que no hubiera falsos negativos o positivos.

Las muestras sometidas a este estudio con sus respectivos controles, se de-parafinaron con xilol, (Entox, Uruguay CAS 1330-20-7) se rehidrataron con alcoholes con graduación decreciente y posteriormente en olla a presión a $126^{\circ} \mathrm{C}$ de temperatura y con solución de recuperación tapón ph 9 , se procedió a la recuperación antigénica, desenmascarando la proteína P 52, a la cual se va a unir el anticuerpo CMV monoclonal mouse (CCH2+DDG9).

Posteriormente, se incubó el anticuerpo en cámara húmeda, a temperatura ambiente y se colocó el kit de conjugación DAKO EnVision-HRP, luego se agregó diaminobencina (DAKO, Canadá, CAS 8007-47-4) para hacer la reacción visible. Entre cada paso del procedimiento se realizó lavados con tampones salinos. Finalmente se realizó una coloración de contraste para diferenciar las áreas de tejido con inmunomarcación de las que no han sido inmunomarcadas, se deshidrató las muestras con alcoholes de graduación creciente, xilol y montaje con Bálsamo de Canadá. (Biopack, proveedor bioquimdiagnostics).

La formalina utilizada es formol buffer tamponado al $4 \%$, con un tiempo de fijación de tres horas.

Se consideró IHQ positiva para CMV a la marcación nuclear en células infectadas por este virus (Figura 1).

\section{Definiciones $^{33-34}$}

Infección por CMV: aislamiento del virus o la detección de antígenos (antigenemia pp 65) o ADN de CMV por técnica calibrada por World Health Organization International Standard for Human CMV en cualquier fluido corporal o tejido.

La hepatitis por CMV según la nueva definición se clasifica en PROBADA como aquella que presenta elementos de disfunción hepática (alteración de las transaminasas, bilirrubinemia, etc.) sumado a la documentación del CMV en: 1) la histopatología (inclusiones intranucleares); 2) IHQ; 3) aislamiento del virus; 4) cultivo rápido o 5) detección por técnica de hibridación por ADN, en ausencia de otra causa identificada de hepatitis.
No se recomienda la categoría de probable ya que su uso lleva confusiones con otras patologías como rechazo del injerto.

\section{Estrategias establecidas para la prevención de infección por CMV}

Universal: La profilaxis universal consiste en la administración de un agente antiviral (valganciclovir-VGC) a pacientes de alto riesgo (donantes positivos/ receptores negativos) durante tres meses.

Selectiva: La profilaxis selectiva consiste en administrar VGC a pacientes de alto riesgo por exposición a timoglobulina durante tres meses.

Estrategia anticipada: Se realiza monitorización de viremia por CMV en forma protocolizada y cuando ésta supera el punto de corte establecido por el laboratorio del centro se inicia VGC para detener su replicación.

Hibrida: Esta estrategia se aplica en pacientes de alto riesgo bajo profilaxis universal o selectiva una vez culminado el antiviral indicado. Consiste en controlar la replicación viral en sangre periférica y reiniciar la terapia si la misma supera el punto de corte establecido por el centro.

El punto de corte establecido en nuestro centro para iniciar estrategia anticipada es de una célula en el antígeno pp 65 (Ag pp65) o carga viral (CV) de CMV de 1.500 $\mathrm{UI} / \mathrm{ml}$.

\section{Análisis estadístico}

Se presentan tablas y gráficos de frecuencia para la descripción de variables cualitativas, así como medidas de resumen para las continuas. El estudio de normalidad de las variables continuas se realizó con test de KolmogorovSmirnov. El análisis de diferencias para las medias se realizó por test $t$ de Student para muestras independientes y para proporciones con test para dos proporciones. La incidencia acumulada se calculó como número de casos con el evento de interés en el total de población en riesgo

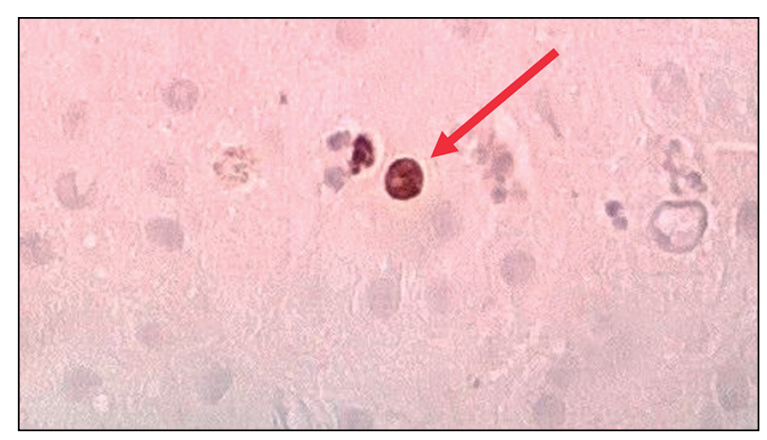

Figura 1. Inmunohistoquímica positiva. Se evidencia la marcación de anticuerpos monoclonales específicos para CMV. 
expresado con una $\mathrm{k}$ de 100. La concordancia entre ambas pruebas se calculó con el Índice Kappa de concordancia. Se fijó un nivel de significación en 0,05. El software estadístico utilizado fue STATA v.12.0.

\section{Ética}

Este estudio fue aprobado por el Comité de Ética de la institución. En todos los casos se solicitó consentimiento informado.

\section{Resultados}

Durante el período de análisis se realizaron 185 TH en este centro. Un total de 41 casos (32 pacientes) cumplían con los criterios de inclusión. De éstos, 27 fueron analizados en la fase retrospectiva y 14 fueron incluidos en la fase prospectiva (Figura 2). La edad promedio fue de $40,0 \pm 2,9$ años estableciéndose mínima y máxima edad, en 15 y 69 años, respectivamente. En relación al género $44 \%(n=14)$ fueron pacientes de sexo femenino. Con respecto al status $\mathrm{CMV}, 97 \%(\mathrm{n}=31)$ presentó un perfil $\mathrm{D}+/ \mathrm{R}+$ (Tabla 1$)$.

Considerando el resultado de la histopatología, 7\% $(n=3$ casos) presentaron elementos específicos de CMV y en $93 \%(n=38)$ se identificaron elementos sugestivos de CMV. El hallazgo más frecuente correspondió a la presencia de microabscesos en $61 \%$ de los casos (Tabla 2).

En seis casos (14,6\%), la IHQ fue positiva para diagnóstico de infección por CMV. En los 17 casos-control se les realizo IHQ siendo la misma negativa en 100\% (Tabla $3)$. Esto traduce un valor predictor negativo (VPN) de $100 \%$ para la histopatología en el diagnóstico de hepatitis por CMV, mientras su valor predictor positivo (VPP) fue de $14,6 \%$.

En los seis casos de hepatitis por CMV, el status CMV fue de riesgo intermedio (receptor + ), en dos de seis casos la histopatología mostró elementos específicos de CMV, mientras que en los restantes cuatro, este estudio mostró elementos sugestivos. (Tabla 4, Figura 3). Tomando a la IHQ como patrón oro, el VPP de la histopatología cuando se identificaron elementos específico fue de $67 \%$, mientras el VPN fue de $89 \%$ (Tabla 4).

Los datos obtenidos muestran un índice de asociación Kappa entre la histopatología y la IHQ de 0,09.

Ochenta y cinco por ciento $(n=30)$ de los casos con IHQ negativa para CMV presentaron diagnósticos alternativos al de hepatitis por CMV, siendo el principal el de rechazo en $47 \%$ (Tabla 5).

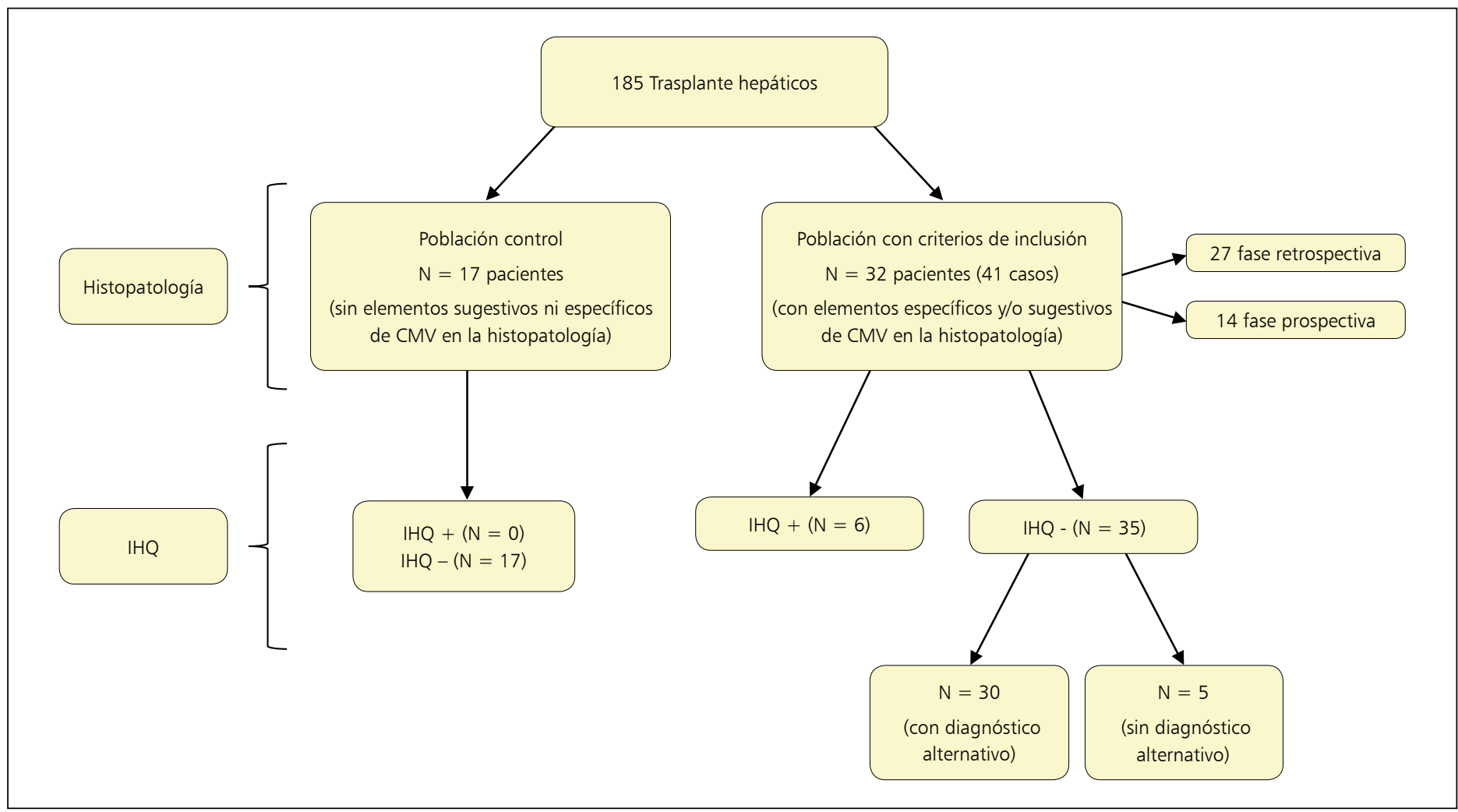

Figura 2. Flujograma de resultados. 
Tabla 1. Generalidades de la población receptora de trasplante hepático con diagnóstico de hepatitis por citomegalovirus según tinción de hematoxilina y eosina: (Unidad Bi Institucional de Enfermedades Hepáticas Complejas (Hospital Militar, Hospital de Clínicas). Uruguay. Período 14 de julio de 2009 a 14 de julio de 2019

\begin{tabular}{lc}
\hline Variables & $\begin{array}{r}\mathbf{n}: \mathbf{4 1} \text { casos } \\
\text { (32 pacientes) }\end{array}$ \\
\hline Edad mediana en años $( \pm D E)$ & $40,0 \pm 2,9$ \\
Sexo femenino $\mathrm{n},(\%)$ & $14(43,75)$ \\
& \\
\hline & \\
Enfermedad que determina falla de órgano & \\
trasplantado $\mathrm{n}(\%)$ & $7(22)$ \\
Cirrosis alcohólica & $11(34,4)$ \\
Autoinmune & $2(6)$ \\
Hepatitis C crónica & $1(3)$ \\
Cirrosis biliar primaria & $3(9,3)$ \\
Nash & $8(25)$ \\
Otras &
\end{tabular}

\section{Status CMV D/R}
$\mathrm{D}-/ \mathrm{R}-$
0
$\mathrm{D}+/ \mathrm{R}+$
$31(96,8 \%)$
$\mathrm{D}-/ \mathrm{R}+$
$1(3,2 \%)$

Estrategia de prevención CMV, n\%

$\begin{array}{lc}\text { Anticipada } & 31(96,8 \%) \\ \text { Universal } & 0 \\ \text { Selectiva } & 1(9,7 \%)\end{array}$

Replicación viral de CMV

CV CMV, media $\pm(D E), n: 36$ pacientes

Ag pp 65, media \pm (DE), n: 22 pacientes

$6 \pm 3$

DE: desviación estándar; CMV: citomagolvirus; D: donante; R: receptor; CV CMV: carga viral de citomegalovirus. 'Otras: Aguda en crónica, Bud Chiari, colangitis esclerosante primaria (CEP), criptogénica, hemocromatosis, hepatitis neonatal, idiosincrática, más de una etiología.
Tabla 2. Histopatología de biopsia hepática por punción, con tinción de hematoxilina y eosina. Unidad Bi Institucional de Enfermedades Hepáticas Complejas (Hospital Militar, Hospital de Clínicas). Uruguay. Período 14 de julio de 2009 a 14 de julio de 2019

$\begin{array}{lc}\text { Histopatología } & \text { n (\%) } \\ \text { Microabscesos } & 17(41) \\ " \text { + pseudoinclusión nuclear } & 1(2,4) \\ " \text { + área de necrosis } & 1(2,4) \\ " \text { + cuerpos apoptóticos + microgranulomas } & 1(2,4) \\ " \text { + cuerpos apoptóticos + infiltrado inflamatorio } & 1(2,4) \\ " \text { + cuerpos apoptóticos } & 1(2,4) \\ " \text { + cuerpos de inclusión intracitoplásmico } & 1(2,4) \\ " \text { + microgranulomas } & 1(2,4) \\ " \text { + microgranulomas + nucleomegalia + cuerpo apoptótico } & 1(2,4)\end{array}$

Granuloma

$1(2,4)$

Microgranulomas

$1(2,4)$

Inclusion citomegalica

$1(2,4)$

Otros $^{1}$

$13(34)$

'Otros: Moderado infiltrado inflamatorio difuso + nucleomegalia; cuerpos apoptóticos + focos necrosis e inflamación lobular; infiltrado inflamatorio mixto, premiación PMN (polimorfonucleares), necrosis focal, PMN; cuerpos apoptóticos + acúmulos PMN; infiltrado PMN lobular.

Tabla 3. Resultados de histopatología, hematoxilina y eosina e inmunohistoquímica en población control y población de estudio. Unidad Bi Institucional de Enfermedades Hepáticas Complejas (Hospital Militar, Hospital de Clínicas). Uruguay. Período 14 de julio de 2009 a 14 de julio de 2019. Resumen de resultados de histopatología e inmunohistoquímica ( $\mathrm{n}: 41$ biopsias hepáticas)

\begin{tabular}{lccc} 
Sugestiva & $\begin{array}{c}\text { Histopatología } \\
\text { Específica } \\
\text { CMV }\end{array}$ & $\begin{array}{c}\text { Negativa para } \\
\text { CMV }\end{array}$ \\
Inmunohistoquímica positiva & 4 & 2 & 0 \\
Inmunohistoquímica negativa & 34 & 1 & 17 \\
Total & 38 & 3 & 17 \\
\hline
\end{tabular}

IHQ: inmunohistoquímica; CMV: citomegalovirus.

\begin{tabular}{|c|c|c|c|c|c|c|}
\hline & Caso 1 & Caso 2 & Caso 3 & Caso 4 & Caso 5 & Caso 6 \\
\hline Edad & 59 & 54 & 21 & 57 & 52 & 60 \\
\hline Sexo & M & M & $\mathrm{F}$ & M & M & M \\
\hline Status CMV & $\mathrm{D}+/ \mathrm{R}+$ & $\mathrm{D}+/ \mathrm{R}+$ & $\mathrm{D}+/ \mathrm{R}+$ & $\mathrm{D}+/ \mathrm{R}+$ & $\mathrm{D}+/ \mathrm{R}+$ & $\mathrm{D}+/ \mathrm{R}+$ \\
\hline CMV viremia & 75.750 & 72.300 & 26.400 & $\begin{array}{c}15 \text { células } x \\
200.000 \text { PMN }\end{array}$ & 52.500 & 23.700 \\
\hline Histopatología & Microabscesos & $\begin{array}{c}\text { Microabscesos, } \\
\text { pesudoinclusión } \\
\text { nuclear }\end{array}$ & Microabscesos & $\begin{array}{l}\text { Inclusiones } \\
\text { citomegálicas }\end{array}$ & $\begin{array}{l}\text { Microabscesos, } \\
\text { áreas de necrosis }\end{array}$ & $\begin{array}{l}\text { Infiltrado inflamatorio } \\
\text { mixto difuso } \\
\text { Nucleomegalia }\end{array}$ \\
\hline
\end{tabular}




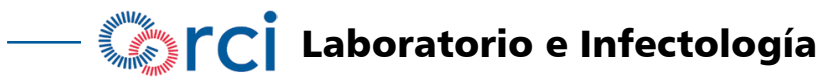

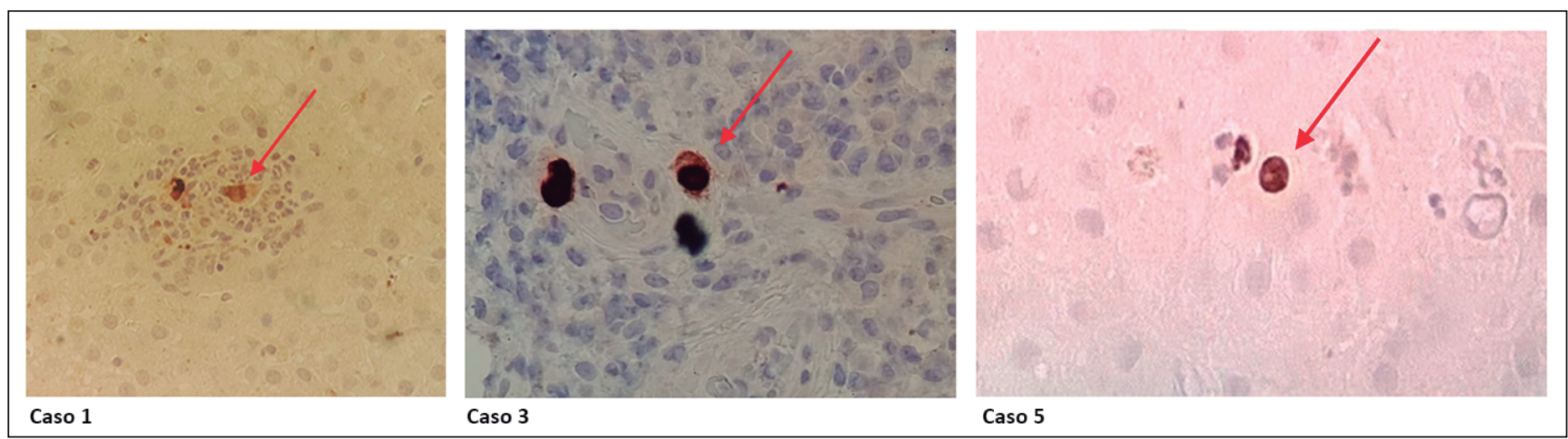

Figura 3. Inmunohistoquímica positiva de casos 1,3 y 5 . Se evidencia la marcación de anticuerpos monoclonales específicos para citomegalovirus.

Tabla 5. Diagnóstico alternativo en pacientes con diagnóstico de hepatitis citomegalovirus por histopatología con tinción de hematoxilina y eosina. Unidad Bi Institucional de Enfermedades Hepáticas Complejas (Hospital Militar, Hospital de Clínicas). Uruguay. Período 14 de julio de 2009 a 14 de julio de 2019. (N: 30)

\begin{tabular}{lcc}
\hline Diagnóstico alternativo & $\mathbf{n}(\%)$ \\
\hline Rechazo & $11(36,7)$ \\
\hline Rechazo + hepatitis autoinmune & $5(16,7)$ \\
\hline Hepatitis de novo autoinmune & $1(3,3)$ \\
\hline Alteración de la arteria hepática & $2(6,7)$ \\
\hline Colangitis & $2(6,7)$ \\
\hline Patología biliar & $1(3,3)$ \\
\hline Daño de preservación & $1(3,3)$ \\
\hline Síndrome linfoproliferativo & $1(3,3)$ \\
\hline Toxicidad farmacológica & $2(6,7)$ \\
\hline Reactivación de VHC & $1(3,3)$ \\
\hline Otra enfermedad viral & $1(3,3)$ \\
\hline Hepatitis por CMV reciente & $1(3,3)$ \\
\hline Esteatosis & $1(3,3)$ \\
\hline
\end{tabular}

VHC: Virus de hepatitis C; CMV: citomegalovirus.

Tabla 6. Características de la replicación viral del citomegalovirus en pacientes con inmunohistoquímica positiva e inmunohistoquímica negativa

\begin{tabular}{llll}
\hline Variables & $\begin{array}{l}\text { IHQ positiva } \\
\mathbf{n}=\mathbf{6}\end{array}$ & $\begin{array}{l}\text { IHQ negativa } \\
\mathbf{n}=\mathbf{3 5}\end{array}$ & Valor $\mathbf{p}$ \\
\hline Carga viral CMV, media $\pm(\mathrm{DE})$ & $50.130,0 \pm 10.989,0$ & $914,1 \pm 549,6$ & $\mathrm{p}=0,011$ \\
\hline Ag pp 65, media $\pm(\mathrm{DE})$ & $13,6 \pm 10,3$ & $3,0 \pm 1,8$ & $\mathrm{p}=0,109$ \\
\hline DE: desviación estándar; IHQ: inmunohistoquímica. & & \\
\hline
\end{tabular}

El promedio del valor de carga viral para CMV en pacientes con IHQ negativa fue 914,1 $\pm 549,6$, mientras que en aquellos con IHQ positiva, el promedio fue 50.130,0 $\pm 10.989,0,(p=0,011)$. Para el Ag pp65 el promedio en pacientes con IHQ negativa fue de $3,0 \pm 1,8$, mientras que en sujetos con IHQ positiva fue 13,6 $\pm 10,3,(p=0,109)$ (Tabla 6, Figura 4). En 100\% de los casos confirmados de hepatitis por CMV $(n=6)$ la viremia detectada fue mayor al punto de corte establecido de $1.500 \mathrm{UI} / \mathrm{ml}$.

Con respecto a la indicación de terapia antiviral por plantearse una hepatitis debida a CMV, a 39\% de los pacientes $(n=16)$ se le administró antiviral. De éstos, 15 recibieron ganciclovir (GCV) como terapia inicial y uno recibió VGC. Si analizamos las dos fases, en la retrospectiva de 27 casos con histopatología sugestiva de CMV se indicó terapia antiviral en $48 \%(n=13)$, mientras que, en la fase prospectiva se indicó esta terapia en $21 \%$ $(\mathrm{n}=3)$ de los casos (pv0,185).

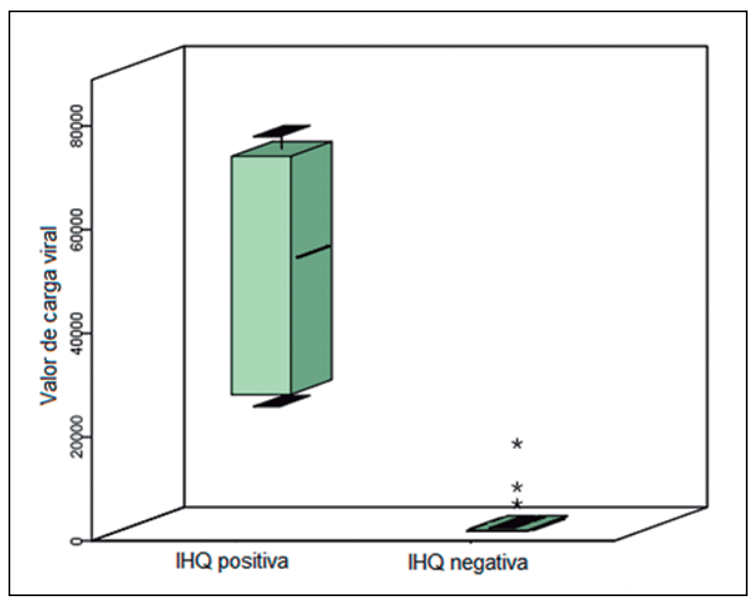

Figura 4. Cargas virales de citomegalovirus discriminadas en pacientes con inmunohistoquímica positiva y negativa. CMV: citomegalovirus; IHQ: inmunohistoquímica. 
contar con técnicas de IHQ para aumentar la especificidad del diagnóstico de hepatitis por CMV en situaciones en que la histopatología es sugestiva. Incluso, al analizar de manera separada los elementos específicos de infección por CMV en la histopatología, comparándolos con la IHQ, el VPP de la histopatología sigue siendo insuficiente, de $67 \%$, mientras el VPN es de $89 \%$. Esto confirma que por sí sola la histopatología no es suficiente para asegurar un diagnóstico adecuado.

Estos resultados concuerdan con lo publicado por otros autores como Barahoke y cols. ${ }^{36}$, y Lautenschlager y cols. ${ }^{37}$, que plantean el uso combinado de técnicas diagnósticas (en este caso la histopatología e IHQ) como una mejor estrategia para optimizar el diagnóstico de hepatitis por CMV en receptores de $\mathrm{TH}$.

El uso de la IHQ como técnica diagnóstica de confirmación determinó una menor incidencia acumulada de hepatitis por CMV en nuestro centro, que fue de 3,2 (6 casos) cada 100 receptores de trasplante. Esta incidencia es inferior a lo reportado por varios programas latinoamericanos $^{32}$ y acorde a lo publicado por centros europeos ${ }^{19}$.

La baja correlación encontrada entre la histopatología y la IHQ en nuestro estudio, consideramos que puede ser atribuida a que los microabscesos fueron el hallazgo identificado en mayor proporción ( $61 \%$ de los casos), mientras que las inclusiones intranucleares, consideradas elementos específicos a nivel de la histopatología, no superaron el $5 \%$ de los casos. Los microabscesos hepáticos se caracterizan por ser un hallazgo sumamente inespecífico que pueden observarse en múltiples complicaciones del $\mathrm{TH}$, como otras enfermedades virales, colangitis, daño de preservación, etc. ${ }^{36-41}$.

Sumado a lo anteriormente planteado, cabe destacar que en $85 \%$ de los casos que presentaron IHQ negativa se identificó un diagnóstico alternativo al de hepatitis por CMV, siendo el principal diagnóstico el rechazo del injerto hepático. Este hecho ya ha sido descrito por otros autores quienes objetivan una incidencia de rechazo de 20 a $40 \%$, principalmente en los primeros meses post trasplante ${ }^{41-46}$. Establecer un correcto diagnóstico entre estas entidades tiene suma relevancia ya que el rechazo del injerto conlleva un tratamiento opuesto al de la hepatitis por CMV (aumento de la inmunosupresión versus disminución de la misma).

Otro pilar utilizado por la mayoría de los centros para el diagnóstico de hepatitis por CMV es la replicación viral en sangre periférica (detección de ADN de CMV o Ag pp65), no existiendo a la fecha un punto de corte estandarizado para definir infección versus enfermedad, lo que constituye una limitante para el uso de esta técnica $^{18,19,47}$. Otras técnicas como la serología carecen de utilidad en el contexto de pacientes inmunodeprimidos receptores de trasplantes para predecir, tanto infección como enfermedad $^{19}$. En el presente estudio, la replica- 
ción viral de CMV en sangre periférica identificada por técnica de ADN, fue significativamente mayor en los casos confirmados por IHQ versus en pacientes con IHQ negativa. Se confirmó, a su vez, la utilidad del punto de corte establecido por nuestro centro de $1.500 \mathrm{UI} / \mathrm{ml}$ para el inicio de terapias antivirales, dado que en $n=0$ de los seis casos confirmados la viremia detectada fue menor al valor de corte determinado.

Finalmente, y como resultado del uso de una técnica confirmatoria con mayor especificidad para el diagnóstico de hepatitis por CMV, en la fase prospectiva logramos una reducción a la mitad del uso de antivirales. Ésta, si bien no fue estadísticamente significativa, probablemente por el tamaño muestral, redunda en un claro beneficio, tanto en términos del propio paciente, como en términos económicos sentando las bases para un nuevo modelo de estudio y diagnóstico de esta complicación.

Planteamos que las limitantes de nuestro trabajo son fundamentalmente el n pequeño de la muestra y la presencia de cinco casos en los que no se llegó a un diagnóstico confirmatorio alternativa. Esto último, pensamos se debe a la ausencia de herramientas diagnósticas para confirmar otros diagnósticos diferenciales como por ejemplo otras etiologías virales en la $\mathrm{PBH}$.
Este es el primer trabajo realizado en nuestro país que documenta el alto VPN de la histopatología, demostrando que cuando ésta no es sugestiva de hepatitis por CMV, podemos descartar de manera razonable este diagnóstico; por el contrario, cuando la histopatología sugiere la presencia de hepatitis por CMV, dado el bajo VPP que presenta, debemos realizar otras técnicas diagnósticas más específicas que la confirmen, como la IHQ.

Los resultados de nuestro trabajo han modificado el estándar de cuidado de los pacientes receptores de TH en nuestro país, realizándoles en la actualidad a todos los pacientes donde la histopatología sugiera hepatitis por $\mathrm{CMV}$, la técnica confirmatoria con IHQ.

\section{Conclusiones}

Reportamos una incidencia acumulada de hepatitis por CMV probada de 3 cada $100 \mathrm{TH}$. La histopatología sin elementos sugestivos y/o específicos de CMV permite descartar razonablemente este diagnóstico. Combinar la histopatología con la IHQ mejoró la certeza diagnóstica en estos casos optimizando las estrategias diagnósticas y terapéuticas.

\section{Referencias bibliográficas}

1.- Crumpacker I I C S, Zhang J L. Citomegalovirus. En Mandell GL, Bennett JE. Dolin R. Eds. Principles and Practice of Infectious Diseases. Philadelphia. Churchill Livingstone. 7th Edition, 2010. p: 1983-2000.

2.- Kotton C N, Kumar D, Caliendo A M, Asberg A, Chou S, Snydman D R, et al; Transplantation Society International CMV Consensus Group. International consensus guidelines on the management of cytomegalovirus in solid organ transplantation. Transplantation.2010; 89 (7): 779-95. doi: 10.1097/TP.0b013e3181cee42f.

3.- Prieto J, Medina J, Menéndez J M, López M, Scalone P, Castelli J, et al. Profilaxis para citomegalovirus. Experiencia del programa de trasplante hepatico. Uruguay, 2013. XXII Congreso Latinoamericano y del Caribe de Trasplante - STALYC / XII Congreso Argentino de Trasplante - SAT. , Buenos Aires (Argentina), 2013.

4.- Zhang L J, Hanpf P, Rutherford C, Churchill W H, Crumpacker C S. Detection of cytomegalovirus, DNA, RNA, and antibody in normal donor blood. J Infect Dis 1995; 171: 1002-6. doi: 10.1093/infdis/171.4.1002.

5.- Smith M G. Propagation in tissue cultures of a cytopathogenic virus from human salivary gland virus (SGV) disease. Proc Soc Exp Biol Med 1956; 92: 424-30. doi: 10.3181/00379727. 92-22498.

6.- Craig J M, Macauley J C, Weller T H, Wirth P. Isolation of intranuclear inclusion producing agents from infants with illnesses resembling cytomegalic inclusion disease. Proc Soc Exp Biol Med 1957; 94: 4-12. doi: 10.3181/00379727-94-22841.

7.- Chee M S, Bankier A T, Becks S, Bohni R, Brown C M, Cerny R, et al. Analysis of the protein coding content of the sequence of human citomegalovirus strain-AD 169. Curr Top Microbiol Immunol 1990; 154: 125-69. doi: 10.1007/978-3-642-74980-3_6.

8.- Cha T, Tom S, Kemble G W, Duke G M, Mocarski E S, Spaete R R. Human citomegalovirus clinical isolates carry at least 19 genes not found in laboratory strains. J Virol 1996; 70: 78-83. doi: 10.1128/JVI.70.1.7883.1996.

9.- Penfold M E, Dairaghi D J, Duke G M, Saederup N, Mocarski E S, G Kemble G W, et al. Cytomegalovirus encodes a potent alpha chemokine. Proc Natl Acad Sci USA 1999; 96 ; 9839-44. doi: 10.1073/pnas.96.17.9839.

10.- Rul P F, Powell K L. Physical and functional interaction of human cytomegalovirus DNA polymerase and its accessory protein (ZCP36) expressed in insect cells. J Virol 1992; 66: 4126-33. doi: 10.1128/JVI.66.7.41264133.1992.

11.- Sullivan V, Talarico C L, Stanat S C, Davis M, Coen D M, Biron K K. A protein kinase homologue controls phosphorylation of ganciclovir in human cytomegalovirus infected cells. Nature 1992; 358: 162-4. doi: 10.1038/358162a0.

12.- Vanarsdall A, Johnson D. Human cytomegalovirus entry into cells. Curr Opin Virol 2012; 2: 37-42. doi: 10.1016/j. coviro.2012.01.001.

13.- Burke H G, Heldwein E E. Correction: crystal structure of the human cytomegalovirus glycoprotein B. PLOS Pathogens 2015; 11 (11): e1005300. https:// journals.plos.org/plospathogens/article/ file?type $=$ printable $\&$ id $=10.1371$ /journal . ppat. 1005300.

14.- Medina J, Aguado J M. Current strategies and future directions in cytomegalovirus (CMV) pneumonitis. In: Pulmonary Infection in the Immunocompromised Patient. USA. Editorial: John Wiley \& Sons; 2009. p.: 383-400.

15.- Medina J, Pérez G, Aguado J M. Interactions between cytomegalovirus and other viruses (HHV6, HHV7, HCV and EBV) in transplantation [Review]. Trends in 
Transplantation 2007; 1: 129-36. https://www. oatext.com/pdf/TiT-1-115.pdf

16.- Kotton C, Kumar D, Caliendo A M, Asberg A, Chou S, Danziger-Isakov L, et al. Updated International Consensus Guidelines on the Management of Cytomegalovirus in SolidOrgan Transplantation. Transplantation 2013; 96: 333-60. doi: 10.1097/ TP.0b013e31829df29d.

17.- Torre-Cisneros J, Aguado J M, Caston J J, Almenar L, Alonso A, Cantisán $\mathrm{S}$, et al. Management of cytomegalovirus infection in solid organ transplant recipients: SET/GESITRA-SEIMC/ REIPI recommendations. Transplant Rev (Orlando) 2016 ; 30 (3): 119-43. doi: 10.1016/j. trre.2016.04.001.

18.- Kotton C N, Kumar D, Caliendo A M, Huprikar S, Chou S, Danziger-Isakov L, et al; The Transplantation Society International CMV Consensus Group. The Third International Consensus Guidelines on the Management of Cytomegalovirus in Solid-organ Transplantation. Transplantation 2018; 102: 900-31. doi: 10.1097/ TP.0000000000002191.

19.- Razonable R R, Humar A. Cytomegalovirus in solid organ transplant recipients-Guidelines of the American Society of Transplantation Infectious Diseases Community of Practice. Clin. Transplant 2019; e13512. doi: 10.1111/ ctr.13512.

20.- Medina J C, Pérez-Sartori G, Caltenco-Serrano $\mathrm{R}$, Aguado J M. Response and pathogenic mechanisms of cytomegalovirus infection in transplant recipients [Review]. Trends in Transplantation 2009; 3: 103-12. https://www. oatext.com/pdf/TiT-3-139.pdf.

21.- Yadav S K, Saigal S, Choudhary N S, Saha S, Sah J K, Saraf N, et al. Cytomegalovirus infection in living donor liver transplant recipients significantly impacts the early posttransplant outcome: A single center experience. Transpl Infect Dis 2018; 20 (4):e12905. doi: 10.1111/tid.12905.

22.- Manuel O, Husain S, Kumar D, Zayas C, Mawhorter S, Levi M E, et al. Assessment of cytomegalovirus specific cell-mediated immunity for the prediction of cytomegalovirus disease in high-risk solid organ transplant recipients: a multicenter cohort study. Clin Infect Dis 2013; 56 (6): 817-24. doi: 10.1093/ $\mathrm{cid} / \mathrm{cis} 993$.

23.- Meesing A, Razonable R R. Pharmacologic and immunologic management of cytomegalovirus infection after solid organ and hematopoietic stem cell transplantation. Expert Rev Clin Pharmacol 2018; 11 (8): 773-88. https://doi.org/ 10.1080/17512433.2018.1501557.
24.- Manuel O, Pang X L, Humar A, Kumar D, Doucette K, Preiksaitis J K. An assessment of donor-to-recipient transmission patterns of human cytomegalovirus by analysis of viral genomic variants. J Infect Dis 2009; 199 (11): 1621-8. doi: 10.1086/598952.

25.- Eid A J, Razonable R R. New developments in the management of cytomegalovirus infection after solid organ transplantation. Drugs 2010; 70 (8): 965-81. doi: 10.2165/10898540000000000-00000.

26.- Fernández-Ruiz $M$, Giménez E, Vinuesa V, Ruiz-MerloT, Parra P, Amat P, et al. Regular monitoring of cytomegalovirusspecific cell-mediated immunity in intermediate-risk kidney transplant recipients: predictive value of the immediate posttransplant assessment.Clin Microbiol Infect 2019; 25 (3): 381.e1-381.e10. doi: 10.1016/j. cmi.2018.05.010.

27.- Gardiner B J, Nierenberg N E, Chow J K, Ruthazer R, Kent D M, Snydman D R. Absolute lymphocyte count: a predictor of recurrent cytomegalovirus disease in solid organ transplant recipients. Clin Infect Dis 2018; 67 (9): 1395-402. doi: 10.1093/cid/ ciy295.

28.- Meesing A, Abraham R, Razonable R R. Clinical correlation of cytomegalovirus infection with CMV-specific CD8+ T-cell immune competence score and lymphocyte subsets in solid organ transplant recipients. Transplantation 2019; 103 (4): 832-8. doi: 10.1097/TP.0000000000002396.

29.- Winston D J, Ho W G, Howell C L, Miller M J, Mickey R, Martin W J, et al. Cytomegalovirus infections associated with leukocyte transfusions. Ann Intern Med 1980; 93: 671-5. doi: 10.7326/0003-4819-93-5-671.

30.- Riverie Marcelin J, Beam E, Razonable R R. Cytomegalovirus infection in liver transplant recipients: Updates on clinical management. World J Gatroenterol 2014; 20 (31): 10658-67. doi: 10.3748/wjg.v20.i31.10658.

31.- Seehofer D, Rayes N, Tullius SG, Schmidt C A, Neumann U P, Radke C, et al CMV hepatitis after liver transplantation: incidence,clinical, course, and long-term follow-up. Liver Transpl 2002;18: 1138-46. doi: 10.1053/ jlts.2002.36732.

32.- Prieto J, Medina J C. Encuesta latinoamericana sobre diagnóstico de citomegalovirus en receptores de trasplante hepático. XXII Congreso Latinoamericano y del Caribe de Trasplante-STALYC. Montevideo. 2017.

33.- Ljungman P, Boeckh M, Hirsh H H, Josephson F, Lundgren J, Nichols G, et al. Definitions of cytomegalovirus infection and disease in transplant patients for use in clinical trials. Clin
Infect Dis 2017; 64(1): 87-91. doi: 10.1093/cid/ ciw668.

34.- Azevedo L S, Pierrotti L C, Abdala E, Costa S F, Strabelli T M, Campos S V, et al. Cytomegalovirus infection in transplant recipients. Clinics (Sao Paulo) 2015; 70 (7): 515-23 doi: 10.6061/clinics/2015(07)09.

35.- Fryer J, Heath A, Minor P D, Collaborative Study Group. A collaborative study to evaluate the proposed 1st WHO International Standard for human cytomegalovirus (HCMV) for nucleic acid amplification (NAT)-based assays. WHO/BS/102138 2010. Geneva, Switzerland: World Health Organization. Biologicals 2016; 44 (4): 242-51. doi: 10.1016/j. biologicals.2016.04.005.

36.- Barkholt L M, Ehrnst A, Veress B. Clinical use of immunohistopathologic methods for the diagnosis of cytomegalovirus hepatitis in human liver allograft biopsy specimens. Scand J Gastroenterol 1994; 29 (6): 553-60. doi: $10.3109 / 00365529409092472$.

37.- Lautenschlager L, Halme K, Höckerstedt Krogerus L, Taskinen E. Citomegalovirus infection of the liver transplant: virological, histological, immunological, and clinical observations. Transpl Infect Dis 2006: 8: 21-30. doi: 10.1111/j.1399-3062.2006.00122.x

38.- Lamps L W, Pinson W, Raiford D, Shyr Y, Scott M A, Washington M K. The significance of microabscesses in liver transplant biopsies: a clinic pathological study. Hepatology 1998; 28 (6): 1532-7. doi: 10.1002/ hep.510280613.

39.- Colina F, Juca N T, Moreno E, Ballestin C, Farinia J, Nevado M, et al. Histological diagnosis of cytomegalovirus hepatitis in liver allografts. J Clin Pathol 1995; 48: 351-7. doi: $10.1136 /$ jcp.48.4.351.

40.- Adeyi O, Fischer S E, Guindi M. Liver allograft pathology: approach to interpretation of needle biopsies with clinicopathological correlation. J Clin Pathol 2010; 63: 47-74. doi: 10.1136/ jcp.2009.068254.

41.- Esquivel C O, Jaffe R, Gordon R D, Iwatsuki S, Shaw B W Jr, Starzl T E. Liver rejection and its differentiation from other causes of graft dysfunction. Semin Liver Dis 1985; 5: 369-74. doi: 10.1055/s-2008-1040634.

42.- Snover D C, Freese D K, Sharp H L, Bloomer J R, Najarian J S, Ascher N L. Liver allograft rejection: an analysis of the use of biopsy in determining the outcome of rejection. Am J Surg Pathol 1987; 11 (1): 1-10. Doi: 10.1097/00000478-198701000-00001.

43.- Busuttil R W, Goldstein L I, Danovitch G M. Liver transplantation today. Ann Intern Med .1986; 104: 377-89. https://doi. org/10.7326/0003-4819-104-3-377. 
44.- Wang Y-C, Wu T-J, Wu T-H, Lee C-F, Chou $\mathrm{H}-\mathrm{S}$, Chan K-M, et al. The risk factors to predict acute rejection in liver transplantation. Transplant Proc 2012; 44: 526-8. doi: 10.1016/j.transproceed.2012.01.041.

45.- García González M, Pera Madrazo C, Bernardos Rodríguez A, Gómez Gutiérrez M, Ignacio Herrero J, Mir Pallardó J, et al. An open, randomized, multicenter clinical trial of oral tacrolimus in liver allograft transplantation: a comparison of dual vs. triple drug therapy. Liver Transpl 2005; 11 (5): 51524. doi: 10.1002/lt.20382.

46.- Doga N, Hüsing-Kabar A, Schmidt H H, Cicinnati VR, Beckebaum S, Kabar I. Acute allograft rejection in liver transplant recipients: Incidence, risk factors, treatment success, and impact on graft failure $\mathbf{J}$
Intern Med Res 2018; 46 (9): 3979-90. doi: $10.1177 / 0300060518785543$.

47.- Preiksaitis J K, Hayden R T, Tong Y, Pang X L, Fryer J F, Heath A B, et al. Are we there yet? Impact of the first international standard for cytomegalovirus DNA on the harmonization of results reported on plasma samples. Clin Infect Dis 2016; 63 (5): 583-9. doi: 10.1093/cid/ ciw370. 